\title{
BMJ Open Management of acromioclavicular joint disease by manual therapy versus corticosteroid injections: the protocol of a non-inferiority study
}

\author{
Alexia Michaut, ${ }^{1}$ Lucie Planche,${ }^{2}$ Lucie Auzanneau, ${ }^{2}$ Grégoire Cormier (1) ${ }^{2}$
}

To cite: Michaut A, Planche L, Auzanneau L, et al. Management of acromioclavicular joint disease by manual therapy versus corticosteroid injections: the protocol of a noninferiority study. BMJ Open 2020;10:e034439. doi:10.1136/ bmjopen-2019-034439

- Prepublication history and additional material for this paper are available online. To view these files, please visit the journal online (http://dx.doi. org/10.1136/bmjopen-2019034439).

Received 19 September 2019 Revised 17 July 2020 Accepted 20 July 2020
Check for updates

(c) Author(s) (or their employer(s)) 2020. Re-use permitted under CC BY-NC. No commercial re-use. See rights and permissions. Published by BMJ.

'Department of Rheumatology, Departmental Hospital Center, La Roche sur Yon, Vendée, France

${ }^{2}$ Departmental of Rheumatology, Vendée Departmental Hospital Center, La Roche sur Yon, France

Correspondence to

Dr Alexia Michaut;

alexia.michaut@chd-vendee.fr

\section{ABSTRACT}

Introduction Degenerative acromioclavicular joint pain accounts for about $4 \%$ of shoulder pain. Various medical and non-medical treatment strategies are available for acromioclavicular joint disease but it is difficult to conduct a comparative evaluation of these treatments. The few studies dealing with the medical management of the disease have conducted no comparative assessment of drug therapies, physiotherapy, joint manipulation and corticosteroid injections. The primary goal of this study is to determine whether manual therapy is not inferior to ultrasound-guided injection of a corticosteroid preparation to decrease acromiocalvicular joint pain at 3 months.

Methods and analysis The acromioclavicular arthropathy managed by manual therapy is a monocentric, comparative, randomised, controlled, non-inferiority study conducted in the Rheumatology Department of Vendée Departmental Hospital, involving two parallel groups receiving either corticosteroid injections or manual therapy. The inclusion criteria are patients who suffer from pain in the shoulder or the proximal part of the arm, with pain located on palpation of the acromioclavicular joint associated with a positive cross-arm test and a positive 0'Brien test. Randomisation will be at a 1:1 ratio. The injection group will receive a single ultrasound-guided injection of $1 \mathrm{~mL}$ of Diprostène and the manual therapy group will receive between one and three sessions at intervals of one per week. The primary outcome will be to compare the Visual Analogue Scale for pain-activity-related score at 3 months for both groups.

Ethics and dissemination The study project has been approved by the appropriate ethics committee (Committee for the Protection of Patients Ouest II in Angers, 30 April 2019, with the registration number of 2019/22). In agreement with current French regulations, signed informed written consent will be obtained from each patient. Results of the main trial and of the secondary endpoints will be submitted for publication in a peerreviewed journal.

Trial registration number NCT03951480.

\section{INTRODUCTION}

This paper was written in accordance with the Standard Protocol Items: Recommendations for Interventional Trials guidelines. ${ }^{1}$
Strengths and limitations of this study

This study is a randomised and controlled study.

- It is the first study comparing corticosteroid injections and joint manual therapy for acromioclavicular joint disease.

- Manual therapy is a topic regularly mentioned by patients and little known by physicians.

- The non-blinded patient and the monocentric aspect are limitations of the study design.

Shoulder pain is a widespread complaint in the general population, with an annual prevalence ranging from $5 \%$ to $47 \% .^{2}$ Acromioclavicular (AC) joint pain accounts for about $4 \%$ of shoulder pains and can be identified on clinical examination. ${ }^{2}$ Degenerative AC joint disease occurs most commonly after the age of 50 years and is more frequent in men in this age group. ${ }^{3}$ Diagnosis is made on the basis of history taking and clinical examination with specific tests such as the cross-arm test. It is positive if by a cross adduction and horizontal adduction with hand to the top of the opposite shoulder the pain is located to the AC joint. The O'Brien test is specific, if the pain is located in the AC joint during anterior flexion, adduction and maximal internal rotation against resistance. ${ }^{4}$ An X-ray of the centre of the AC joint should then be taken to confirm degenerative joint disease. In their literature review, Chaudhury ${ }^{5}$ report that it is difficult to conduct a comparative evaluation of the various medical and nonmedical treatment strategies for AC joint pain because these strategies cannot be compared in a blinded fashion. The few studies dealing with the medical management of the disease conducted no comparative assessment of drug therapies, physiotherapy, joint manipulation or corticosteroid injections. ${ }^{5}$

The Visual Analogue Scale (VAS) to measure pain at rest, pressure pain and night-time 
pain is often used. ${ }^{6}$ Pain VAS is the most commonly used method to rate a patient's subjective assessment of pain. It has good reproducibility, is able to track pain progression in the same patient and is easy to use even in patients with cognitive impairment. ${ }^{8}$ In one study of Sabeti-Aschraf ${ }^{6}$ after a treatment by one infiltration, the VAS pressure pain score progressed from $7.8 \pm 1.99$ at T0 to $5.9 \pm 3.41$ at T2 after 2 weeks of treatment $(\mathrm{p}<0.0013)$, while the pain at rest score went from $6.9 \pm 1.57$ at T0 to $5.8 \pm 2.25$ at T2 $(\mathrm{p}<0.0213)$. In a second study of SabetiAschraf ${ }^{7}$ the VAS pressure pain score progressed from $5.76 \pm 2.00$ to $2.68 \pm 2.34$ while the night-time score went from $5.22 \pm 2.27$ to $2.42 \pm 2.31$ after 3 weeks of treatment, irrespective of the mode of injection $(\mathrm{p}<0.0001)$.

There are several shoulder function tests, including the Constant Score, ${ }^{9}$ which was used in the studies referred to above but which has not been validated in a French version. It is difficult to perform in daily practice, as it involves the measurement of angles of joint amplitudes that are of interest to surgeons but that are not useful for our study. The Disabilities of the Arm, Shoulder and Hand (DASH) questionnaire ${ }^{10}$ is specifically used to assess the functional disability of patients during leisure activities and in the workplace. The test is widely used in medical studies, in particular for shoulder injections for pain management. The questionnaire is easy to perform, does not require any joint measurements and has been validated in a French-language version. The Oxford Shoulder Score is another patient-based questionnaire, which is significantly correlated with the Constant Score but is easier to implement. ${ }^{11}$ The results help guide the choice of either medical or surgical management. This evaluation, however, falls outside the scope of our study. The Specific AC Score, a new assessment tool validated in English by surgeons, is too recent to have been compared with other methods. ${ }^{12}$

Regarding ultrasound-guided injections, in the study of Park ${ }^{13}$ patients with shoulder pain had a better functional outcome after 3 months and 6 months with ultrasoundguided injections. The findings suggest that ultrasoundguided injections are more effective.

Performed by an experienced physician, manual therapies could act as a complement to conventional medicine for mild joint pain such as that associated with osteoarthritis. They could be an alternative to injections because the study of Rhon reported transient pain with injections. ${ }^{14 a}$ Harris ${ }^{15 a}$ Winters ${ }^{16}$ compared corticosteroid injections with physiotherapy (in the form of massages and self-administered exercises) and with passive movement and joint manipulation performed by a manual therapist for shoulder complaints. The duration of pain was considerably shorter after manipulation as compared with physiotherapy. Almost $70 \%$ of patients in the manipulation group considered themselves cured compared with only $10 \%$ of those who had received physiotherapy $(p<0.001)$. In addition, manipulation was associated with less therapeutic failure as compared with physiotherapy. In patients with synovitis of the shoulder, the duration of symptoms was shorter and therapeutic failure was far less often reported after corticosteroid injections than it was after manipulation or physiotherapy. In this study, the cause of the pain was not taken into consideration, with the authors only indicating the presence (or lack) of synovitis. It seems reasonable to propose manipulation in the management of cases of shoulder pain without synovial involvement, as in AC osteoarthritis.

In the literature review of Peek ${ }^{17}$ the cited randomised controlled trials analysed thoracic manual therapies in comparison to conventional treatments such as analgesics, non-steroid anti-inflammatory drugs (NSAIDs), corticosteroid injections. ${ }^{15}$ determined the short-term and long-term effects of manual therapy management of patients with non-acute AC joint pain. After six sessions of manual therapy, significant short-term and long-term improvement in pain and disability was observed. Assessments were made on the basis of Shoulder Pain and Disability Index scores and the American Shoulder and Elbow Surgeon Scale. The pain VAS was not used. All these studies point towards some beneficial effects of manual therapy for the management of pain and disability in the shoulder. However, they involved small patient sample sizes, gave no details about the initial shoulder complaint and did not compare the effects of manual therapy and injections.

\section{Objectives}

\section{Main objective}

Comparing the medium-term efficacy of manual therapy management of pain in degenerative $\mathrm{AC}$ joint disease with that of ultrasound-guided injection of a corticoid preparation (Diprostene) is the main objective.

\section{Secondary objectives}

- Progression of pain at rest and of night-time and activity-related pain over the 7 days before visits at day 0 (D0), month 1 (M1) and month 3 (M3). Recording of the mean and greatest pain during the preceding week.

- Progression of pain at rest at visits at D0, M1, and M3 and of activity-related pain at visits at D0 and M1.

- Progression of upper-limb-related quality of life as assessed by the DASH self-administered questionnaire at D0, M1 and M3.

- Assessment of the clinical tolerance of manipulations compared with that of injections on the basis of pain VAS scores at D0.

- Assessment of the patient's perception of manipulations compared with that of injections at $\mathrm{D} 0$ on the basis of replies to three questions, two before the session and one after:

Which procedure do you think will be more painful? Which do you think will be more effective? Would you recommend the procedure that you underwent to a relative or friend?

- Assess the patient's perception of disease progression. 
- Describe the adverse effects and events reported at D0, M1 and M3 (booklet).

- Record the consumption of analgesics and NSAIDs between D0 and M3 (booklet).

\section{Methods}

\section{Study design}

It is a monocentric, comparative, randomised, controlled non-inferiority study involving two parallel groups receiving either corticosteroid injections or manual therapy.

The study was conducted in the Rheumatology Department of the Vendée Departmental Hospital (CHD), La Roche sur Yon, France.

\section{Inclusion criteria}

- Patients aged between 40 and 70 years.

- Pain in the shoulder or the proximal part of the arm with pain located on palpation of the $\mathrm{AC}$ joint+positive cross-arm test+positive O'Brien test.

- The absence of pain or $<4$ score on Hawkins test.

- Symptomatic AC osteoarthritis confirmed by X-ray.

- Pain at rest or activity-related pain (VAS $\geq 4$ ) with a duration of longer than 3 months.

- Patient able to understand the protocol and give informed consent.

- Patient with social security coverage.

NSAIDs or oral analgesics can be taken during the study period.

\section{Exclusion criteria}

- Contraindication to scapular or cervical manipulation.

- Contraindication to injections (unbalanced diabetes with $\mathrm{HbA1c}>8.5 \%$, unbalanced hypertension and current infection).

- Recent trauma of the AC joint $<6$ months.

- Local or generalised infection.

- Severe bleeding disorder and current anticoagulant therapy (new oral anticoagulants, antivitamin $\mathrm{K}$ and clopidogrel).

- Severe and/or uncontrolled hypertension.

- Unbalanced diabetes.

- Live vaccines.

- Known allergy to Diprostene, including its excipients (methyl parahydroxybenzoate, propyl parahydroxybenzoate and benzyl alcohol).

- Inflammatory rheumatism (rheumatoid arthritis, polymyalgia rheumatica and ankyloses spondylitis).

- Microcrystalline arthritis of the shoulder (gout or calcium pyrophosphate deposition disease).

- History of surgery or of a shoulder injury requiring a surgical procedure or arthroscopic intervention.

- Previous injections within the last 6 months.

- Severe water and/or sodium retention (hypernatremia), particularly in cases of heart failure, decompensated liver failure (oedema and ascites) and severe renal failure.

- Immunocompromised or haemodialysis patients.
- Pregnant or breastfeeding women.

- Patients under guardianship or curatorship or deprived of liberty.

- Patients participating in another interventional clinical research study involving a drug or medical device.

- Patients whom the investigator considers unable to comply with the study protocol.

- Patients with a diagnosis of associated fibromyalgia.

\section{Intervention}

The inclusion and exclusion criteria will be checked during the enrolment visit. The aims and protocol of the study will then be explained to eligible patients, who will be given oral and written information and asked to sign a consent form (cf online supplemental file). During this consultation, the groups will be randomised so as to schedule in advance the sessions for the group allocated to manual therapy. Randomisation will be done through the Ennov Clinical website according to a ratio of 1:1.

Depending on the group to which they have been allocated, patients will receive either a single ultrasound-guided injection of $1 \mathrm{~mL}$ of Diprostene or manual therapy management comprising three sessions of $30 \mathrm{~min}$ each at intervals of 1 week. In line with usual practice, ${ }^{14}$ it will be possible to halt these sessions after assessment of clinical progress by the therapist. If the VAS score is $<2$ for activity-related pain, it will be rated as not painful or mildly painful and manual therapy will be discontinued.

The manual therapy was performed by only one rheumatologist practicing in public hospital, with 5 years of manual medicine training and having experience of 7 years of practice. It consists of performing contractions and isometric stretchings of the shoulder and neck muscles (deltoid, scapula elevator, trapezoid, scalens, sternocleidomastoid muscles and splenius), inhibition of muscle trigger points and mobilisation of the AC joint.

This joint and muscle work are performed after a phase of evaluation of muscle contractures by shoulder and cervical muscle palpation and joint amplitudes of the shoulder. The evaluation is comparative to the control side. Evaluation of trigger points corresponds to a pain triggered by a pressure of $100 \mathrm{~g}$ in muscles in preferential area in the muscles described by Travell and Simons. ${ }^{18}$ The evaluation of muscle corresponds to palpation of muscular fibres, muscular contractures and stretch pain.

Manual therapy does not include cervical manipulations. The mobilisations of the ACjoints are the same in all patients. The patient sits or is in dorsal decubitus. The mobilisation of the joint is done by lowering the clavicular, mobilisation of the joint backwards in a sitting position (acromion is brought back) and mobilisation with internal rotation of the shoulder with arm at $90^{\circ}$ of abduction.

The muscular work will depend on the contractures found at the initial clinical examination and consist in contraction and isometric stretching, resting, sliding pressure and deep transversal massage on the muscle, that is to say that we perform stretching and relaxation on the muscles of 
the shoulders, in particular on those which are particularly contracted.

Ultrasound-guided injection of corticosteroids will be performed under strict aseptic conditions after five applications of Betadine, skin disinfectant, or an equivalent product for allergic patients. Injections of $1 \mathrm{~mL}$ will be performed in accordance with good clinical practice under ultrasound guidance by a designated state-registered nurse. Dose quantity will not be modified, as the product is administered in a single injection. Local injections of corticosteroids can unbalance diabetes, a psychotic state and severe hypertension. They should be administered with caution to patients at a high risk of infection, in particular, those fitted with a prosthetic implant. The risk of a vasomotor reaction and, in particular, of thoracic pain should be taken into consideration in patients with underlying progressive cardiovascular disease. Care should be taken to not perform intratendinous injections because of the risk of rupture, nor to use intravenous and intramuscular routes. This product cannot be inhaled by nebuliser.

Follow-up will be adapted according to the randomisation $\mathrm{arm}$. In the experimental arm, patients will be given between one and three sessions of manual therapy at D0, week 1 (W1) and week 2 (W2) according to pain progression. They will have a follow-up consultation at M1 after the first session with a clinical research nurse dedicated to this consultation (there is only one nurse who will perform this consultation) and at M3 after the first session with a doctor (several consultants will be able to carry out this consultation, that is to say the doctors dedicated to clinical research trained in this study, there are seven doctors trained). The different examinations, scores and assessment data will be recorded during the different visits according to the schedule listed in table 1. Assessment of the main criterion at M3 (VAS activity-related score) will be recorded by a medical evaluator different from the physician who performed the manual therapy so as to maintain blinding of the randomisation arm.

In the control arm, patients will receive a single injection of corticosteroids (Diprostene) at D0. They will then be seen at a follow-up consultation at M1 post-injection by a clinical research nurse and at M3 post-injection by a medical assessor. The different examinations, scores and assessment data will be recorded during the different visits according to the schedule listed in table 1. Assessment of the main criterion at M3 (VAS activity-related score) will be recorded by a medical evaluator different from the physician who administered the injection so as to maintain blinding of the randomisation arm.

\section{Patient and public involvement}

There was no patient or public involvement in this study.

\section{Outcomes}

Main assessment criteria

The main assessment criteria will be the VAS activityrelated score of the patient at M3.

\section{Table 1 Study time frame}

\begin{tabular}{|c|c|c|c|c|c|c|}
\hline Actions & $\begin{array}{l}\text { D30 } \\
\text { Inclusion }\end{array}$ & $\begin{array}{l}\text { D0 } \\
\text { Infiltration or } \\
\text { manipulation }\end{array}$ & $\begin{array}{l}\text { W1 } \\
{ }^{*} \text { Exp. arm }\end{array}$ & $\begin{array}{l}\text { W2 } \\
{ }^{\star} \text { Exp. arm }\end{array}$ & W4 (M1) & $\begin{array}{l}\text { W12 (M3) } \\
\text { Final } \\
\text { assessment }\end{array}$ \\
\hline Patient information & $x$ & & & & & \\
\hline Check inclusion/exclusion criteria & $\mathrm{x}$ & & & & & \\
\hline Sign informed consent & $x$ & & & & & \\
\hline Randomisation & $x$ & & & & & \\
\hline Clinical examination & $x$ & $x$ & & & & \\
\hline $\begin{array}{l}\text { Paraclinical examinations } \\
\text { AC X-ray }\end{array}$ & $x$ & & & & & \\
\hline $\begin{array}{l}\text { Efficacy and/or safety measurement } \\
\text { Pain VAS procedure }\end{array}$ & & $x$ & $X^{*}$ & $X^{*}$ & & \\
\hline Verbal scale overall assessment & & & & & $\mathrm{X}$ & $x$ \\
\hline Concomitant therapies & & $x$ & & & $x$ & $x$ \\
\hline Therapies: Diprostene injection & & $x$ & & & & \\
\hline Manipulations & & $x$ & $x$ & $x$ & & \\
\hline Adverse events & & $X$ & $\mathrm{X}$ & $\mathrm{X}$ & $\mathrm{X}$ & $X$ \\
\hline
\end{tabular}

AC, acromioclavicular; D0, day 0; D30, day 30; DASH, Disabilities of the Arm, Shoulder and Hand; M1, month 1; M3, month 3; VAS, Visual Analogue Scale; W1, week 1; W2, week 2; W4, week 4; W12, week 12. 


\section{Secondary assessment criteria}

- The mean and maximum VAS scores of pain at rest and night-time and activity-related pain during the 7 days preceding the visits at D0, M1 and M3 will be recorded.

The VAS is a self-administered test that allows patients to assess their perceived pain on a rating scale that goes from 0 (no pain) to 10 (pain as bad as it could be). ${ }^{19}$

A verbal scale is a dynamic criterion that assesses disease progression in relation to a given reference. It is officially recommended for use as a measurement tool in research projects on arthrosis. Numerous trials have shown it to be effective. $^{19}$

- VAS scores of pain at rest will be recorded during the visits at D0, M1 and M3 and those of activity-related pain during the visits at D0 and M1.

Activity-related pain corresponds to the greatest pain felt on movement of the shoulder during abduction, adduction and anteflexion.

Pain at rest corresponds to the pain felt when the upper limb is in a neutral position and relaxed.

- Scores from the DASH questionnaire, which assesses functional disability during daily and leisure activities and in the workplace, will be recorded at D0, M1 and M3. ${ }^{20}$

The DASH questionnaire provides a functional score for the upper limbs, including the shoulder. It assesses patient health status in the previous week and has been validated in a French-language version. It is a 30-item, selfreport questionnaire focusing on the difficulty involved in performing various physical activities. The scores range from 0 to 100 and are calculated on the basis of ( (addition of answers) -1$) \times 25$.

The score can be validated only if the patient has replied to $90 \%$ of the questions (ie, three missing values at most).

- Patient assessment of the pain felt during the procedure at the end of the consultation: VAS pain score after the intervention.

- Verbal scale assessment of overall patient progression at M1 and M3.

- Record of the adverse effects and events of the two management therapies.

- Patient assessment of analgesic consumption during the week before the consultation and throughout follow-up (follow-up booklet).

- Assessment of patients' perception of the procedure before injection or manipulation. Which one do they think will be more painful and which one do they think will be more effective? Would they recommend the procedure they underwent to a relative or friend?

- During the clinical follow-up visit at 3 months, the patient will be asked the following question:

In comparison to the beginning of the therapy (D0), do you consider that today your shoulder complain:

- has gotten worse,

- is stable or not improved,

- shows modest improvement,

- shows mild improvement,
- has greatly improved, or

is cured?

\section{Duration of participation}

The patient participation schedule is listed in table 1 .

\section{Sample size}

The study is a non-inferiority trial on the decrease in activity-related pain at 3 months as assessed by the difference in the score measured from 3 months to baseline between the groups. In shoulder complaints, a difference of 1 in the VAS score (0-10) can be considered clinically significant (Hawker, 2011). In our study, therefore, we decided to set the zone of non-inferiority at 1 on the VAS. Alpha and beta risks were set at $2.5 \%$ and $20 \%$, respectively. We assumed that the common SD between the two groups would be 2 . On the basis of these different hypotheses, it was calculated that 128 patients would be required for the study. To guarantee the study's statistical power, $5 \%$ extra patients will be randomised, making an overall total of 136 patients.

\section{Recruitment}

All patients who were seen in or referred to the Rheumatology Department of the Vendée Departmental Hospital (CHD), France, for AC osteoarthritis and who fulfilled the inclusion criteria of the study were invited to follow the protocol. The patient inclusion period is 30 months.

\section{Allocation of the interventions \\ Randomisation:}

Randomisation will be on a 1:1 ratio basis and performed in blocks with Ennov Clinical software: https://nanteslrsy.hugo-online.fr/EnnovClinical/. Connection will be made by login, a password and a study number provided by the data manager of the Research Unit of the CHD of La Roche sur Yon. The investigator will perform randomisation after checking patients' eligibility to take part in the study and during the visit at D30. The inclusion number will be assigned automatically at the time of randomisation. Confirmation will be sent by email to the person who did the randomisation and to all other individuals concerned.

The randomisation list will be created by the statistician of the Research Unit of the CHD of La Roche sur Yon. An explanatory guide to the randomisation will be available online at Ennov Clinical website.

\section{Blinding}

The patients will not be blinded to group assignment because they and the physicians will not be able to distinguish between the two treatment methods. The physician assessor of the main criterion at M3 will be blinded to the therapy used.

\section{Data collection}

An electronic Case Report Form (eCRF) will be created for each patient. All the information required for the protocol will be recorded in the eCRF. This should include 
the data that confirm compliance with the protocol and all the data needed for statistical analyses. The information will indicate any major deviation from the protocol. Any missing data will be coded.

The person(s) overseeing the completion of the eCRFs will be defined and identified in the table of delegation of responsibilities for each centre (kept in the investigator's file).

The investigator is responsible for the exactitude, quality and relevance of all the data entered. In addition, as they are entered, the data will be checked immediately by coherence controls. The investigator should check any changes in the eCRF. The changes will be subject to an audit trail. A justification could be included in the form of a comment.

A database and data entry forms along the lines of the eCRF will be created to collect the clinical data in accordance with the protocol and existing regulations.

\section{Statistical method}

Statistical analyses will be performed using SAS V.9.4 software. As the study is a non-inferiority trial, analyses will be conducted on an intention-to-treat basis according to per-protocol analysis.

Description of the patients is carried out according to the randomisation group on inclusion.

The different variables will be presented in their entirety and by group. The description will include effectives and percentages for qualitative variables and the minimum, maximum, mean, SD and median for quantitative variables.

\section{Analysis of the main criterion}

The VAS activity-related score will be compared in the two groups at 3 months. The $95 \%$ CI for the difference (manipulation vs injection) between the two VAS scores at M3 will be estimated by a mixed linear model, taking into account the repeated nature of the data. The upper limit of the interval of confidence will be the non-inferiority margin defined by 1 point.

\section{Analysis of secondary criteria}

VAS scores for pain at rest and night-time and activityrelated pain will be recorded at D0, M1 and M3. The different scores will be compared with a mixed linear regression model, taking into account the repeated nature of the data.

Quality of life will be compared on the basis of DASH questionnaire scores recorded on D0, M1 and M3. Score progression will be assessed using a mixed linear regression model, taking into account the repeated nature of the data.

Patient perception and feelings during the procedure will be assessed on the basis of a VAS score just after the intervention. The scores of the two groups will be compared using Student's t-test.

The patient's perception of the disorder will be assessed on a verbal scale at M1 and M3. Score progression will be assessed with a mixed linear regression model, taking into account the repeated nature of the data.
Consumption of analgesics will be recorded throughout the study period up to M3. Overall consumption will be assessed using a linear regression model.

\section{Data monitoring}

Before the beginning of patient recruitment, the health professionals involved in the study will attend a training session that explains the protocol and how to record the data entered in the eCRF. The eCRF is a secured, interactive tool that, in our study, will be managed by the hospital's Promotion Department of the Vendée CHD, La Roche sur Yon. Clinical research physicians and staff will be in charge of patient screening. The patients will be enrolled in the study by a physician in compliance with the rules of the protocol and data collection.

The study will be conducted within the framework of the Méthodologie de Référence (Reference Methodology) MR-001, in conformity with the French Data Protection Law, in application of article 54, paragraph 5 of law $\mathrm{n}^{\circ} 78-17$ of 6 January 1978 updated (Deliberation no 2016-262 of 21 July 2016).

The Vendée CHD, La Roche sur Yon, as the promoter of the study, has signed a commitment to comply with the Reference Methodology.

\section{Prejudice}

A patient can be temporarily withdrawn from the trial by the physician in charge if there is suspicion of a serious adverse effect attributable to the product administered during the study. In this event, and in accordance with French regulations, a specific procedure has been drawn up and can be consulted in the study protocol.

\section{Audit}

The Promotion Department of the Clinical Research Unit of the Vendée CHD performs regular audits of data collection (screening and clinical data).

\section{ETHICS AND DISSEMINATION}

\section{Approval of the ethics committee}

The clinical trial will be conducted in accordance with the current version of the Declaration of Helsinki and good clinical practice guidelines. The project received approval from the local Ethics Committee for the Protection of Patients (Quest II) in Angers, 30 April 2019, with the registration number of 2019/22.

\section{Consent or assent}

In accordance with existing French regulations, written consent to participate in a trial is required from the patient. The patient will be asked to give informed consent at the preenrolment visit at D30 and will provide the signed document to the physician on inclusion. Should the patient decline to give consent, the physician undertakes to treat the patient in accordance with existing regulations. A patient can withdraw from the study at any time without incurring any liability or prejudice as a result, and without any effect on the quality 
of care provided. In this event, the patient must inform the physician of his or her decision.

In the event of withdrawal of consent, personal data already collected will be processed using a computer unless written opposition is received.

\section{Confidentiality}

The study will be conducted in accordance with the guidelines of the French Data Protection Authority and general data protection regulations. All source data will be kept at the investigating centre and the coordinating centre for data management. The electronic database will be anonymous and kept for 15 years according to good clinical practice.

\section{Conflicts of interest}

None of the physicians taking part in the study have conflicts of interest, financial or otherwise, related to the conduct of the study. The MSD Laboratories, France, which market Diprostene, at no time took part in the development of the protocol design and will not intervene in any stage of the study (data collection, analysis and interpretations, and decision to publish).

\section{Access to data}

Only the coordinating physician and the hospital's Promotion Department of the Vendée CHD will have access to the final data for analysis. The physician investigators will by then have signed a no-access contract.

\section{Communication}

The coordinator of the study is committed to communicating the results of the study to the investigators and participants who ask to be informed. The results will be communicated only after the end of the study, after the last visit of the last patient or in the event of premature termination of the trial. The results will be reported to health professionals and groups of experts via publications and oral communications or posters at medical congresses.

Practical arrangements concerning rules for publication will be established in agreement with the physician investigators and other participants in the study.

\section{Acknowledgements Jeffrey Watts for translation.}

Contributors AM, GC, LA and LP wrote the manuscript and took part in the study design. LP wrote the statistical analysis plan and estimated the sample size. AM, GC, LA and LP obtained funding for the study. All the authors contributed to data collection, made a critical reading of the manuscript and read and approved the final version.

Funding This study was supported by a grant from CHD Vendée in response to an internal call for bids.

Competing interests None declared.

Patient consent for publication Not required.

Provenance and peer review Not commissioned; externally peer reviewed.

Supplemental material This content has been supplied by the author(s). It has not been vetted by BMJ Publishing Group Limited (BMJ) and may not have been peer-reviewed. Any opinions or recommendations discussed are solely those of the author(s) and are not endorsed by BMJ. BMJ disclaims all liability and responsibility arising from any reliance placed on the content. Where the content includes any translated material, BMJ does not warrant the accuracy and reliability of the translations (including but not limited to local regulations, clinical guidelines, terminology, drug names and drug dosages), and is not responsible for any error and/or omissions arising from translation and adaptation or otherwise.

Open access This is an open access article distributed in accordance with the Creative Commons Attribution Non Commercial (CC BY-NC 4.0) license, which permits others to distribute, remix, adapt, build upon this work non-commercially, and license their derivative works on different terms, provided the original work is properly cited, appropriate credit is given, any changes made indicated, and the use is non-commercial. See: http://creativecommons.org/licenses/by-nc/4.0/.

ORCID iD

Grégoire Cormier http://orcid.org/0000-0002-8852-3687

\section{REFERENCES}

1 Chan A-W, Tetzlaff JM, Gøtzsche PC, et al. SPIRIT 2013 explanation and elaboration: guidance for protocols of clinical trials. BMJ [Internet]..

2 Juel NG, Natvig B. Shoulder diagnoses in secondary care, a one year cohort. BMC Musculoskelet Disord 2014;15:89.

3 Mall NA, Foley E, Chalmers PN, et al. Degenerative joint disease of the acromioclavicular joint: a review. Am J Sports Med 2013;41:2684-92.

4 Saccomanno MF, DE leso C, Milano G. Acromioclavicular joint instability: anatomy, biomechanics and evaluation. Joints 2014;2:87-92.

5 Chaudhury S, Bavan L, Rupani N, et al. Managing acromio-clavicular joint pain: a scoping review. Shoulder Elbow 2018;10:4-14.

6 Sabeti-Aschraf M, Stotter C, Thaler C, et al. Intra-articular versus periarticular acromioclavicular joint injection: a multicenter, prospective, randomized, controlled trial. Arthroscopy 2013;29:1903-10.

7 Sabeti-Aschraf M, Ochsner A, Schueller-Weidekamm C, et al. The infiltration of the ac joint performed by one specialist: ultrasound versus palpation a prospective randomized pilot study. Eur $J$ Radiol 2010;75:e37-40.

8 Heller GZ, Manuguerra M, Chow R. How to analyze the visual analogue scale: myths, truths and clinical relevance. Scand J Pain 2016;13:67-75.

9 Chelli M, Levy Y, Lavoué V, et al. The "auto-constant": can we estimate the constant-murley score with a self-administered questionnaire? A pilot study. Orthop Traumatol Surg Res 2019;105:251-6.

10 HAS. EPAULE_SCORE_DASH.pdf. Available: https://www.s-f-t-s. org/images/stories/documentations/EPAULE_SCORE_DASH.pdf [Accessed 26 Sept 2018].

11 Tuton D, Barbe C, Salmon J-H, et al. Transcultural validation of the Oxford shoulder score for the French-speaking population. Orthop Traumatol Surg Res 2016;102:555-8.

12 Barwood SA, French JA, Watson LA, et al. The specific AC score (sacs): a new and validated method of assessment of isolated acromioclavicular joint pathology. J Shoulder Elbow Surg 2018;27:2214-23.

13 Park KD, Kim TK, Lee J, et al. Palpation versus ultrasound-guided acromioclavicular joint intra-articular corticosteroid injections: a retrospective comparative clinical study. Pain Physician 2015;18:333-41.

14 Rhon DI, Boyles RB, Cleland JA. One-year outcome of subacromial corticosteroid injection compared with manual physical therapy for the management of the unilateral shoulder impingement syndrome: a pragmatic randomized trial. Ann Intern Med 2014;161:161-9.

15 Harris KD, Deyle GD, Gill NW, et al. Manual physical therapy for injection-confirmed nonacute acromioclavicular joint pain. J Orthop Sports Phys Ther 2012;42:66-80.

16 Winters JC, Sobel JS, Groenier KH, et al. Comparison of physiotherapy, manipulation, and corticosteroid injection for treating shoulder complaints in general practice: randomised, single blind study. BMJ 1997;314:1320-5.

17 Peek AL, Miller C, Heneghan NR. Thoracic manual therapy in the management of non-specific shoulder pain: a systematic review. $J$ Man Manip Ther 2015;23:176-87.

18 Shah JP, Thaker N, Heimur J, et al. Myofascial trigger points then and now: a historical and scientific perspective. Pm R 2015;7:746-61.

19 Haefeli M, Elfering A. Pain assessment. Eur Spine J 2006;15:S17-24.

20 HAS. EPAULE_SCORE_DASH.pdf+. Available: https://www.s-f-t-s. org/images/stories/documentations/EPAULE_SCORE_DASH.pdf [Accessed 8 Jan 2020]. 